Check for updates

Cite this: Mater. Adv., 2021, 2, 440

Received 2nd September 2020 Accepted 30th November 2020

DOI: $10.1039 / \mathrm{d} 0 \mathrm{ma00669f}$

rsc.li/materials-advances

\title{
Effect of heparin and peptide conjugation on structure and functional properties of alginate in solutions and hydrogels $\dagger$
}

\author{
Nir Goldberg, ${ }^{a}$ Yulia Shmidov, ${ }^{a}$ Olga Kryukov, ${ }^{b}$ Dina Aranovich, ${ }^{a}$ Smadar Cohen $^{\mathrm{b}}$ \\ and Ronit Bitton (iD) *ac
}

\begin{abstract}
Alginate decoration with more than one type of biomolecule is becoming a prevalent process in the pursuit to turn alginate hydrogels into synthetic extracellular matrices (ECMs). Here we present a systematic study of the structural-physical properties-function relationship of alginate- $G_{4} R G D Y$-heparin aqueous solutions and hydrogels. Small-angle X-ray scattering (SAXS) and rheology were used to characterize the systems' nanostructure, viscosity of the solutions, and storage modulus of the hydrogels. The bioactivity of these gels was explored by evaluating their ability to sustain the release of vascular endothelial growth factor (VEGF). We show that the mode and order of conjugating the heparin and the peptide to the alginate backbone greatly affect the modified alginate hydrogels' structure-properties-function relations. Moreover, we show that a detailed structural analysis of the conjugated architecture in solution can be used as a tool to adapt the properties of alginate-heparin-peptide hybrid hydrogels.
\end{abstract}

\section{Introduction}

Alginate is a linear, hydrophilic, polysaccharide biopolymer consisting of $\beta$-D mannuronic and $\alpha$-L guluronic acid blocks, which occur in the cell walls of brown seaweed. ${ }^{1}$ Alginate hydrogels have been proven to be promising scaffolds for tissue regeneration and repair $^{2-4}$ for bone, ${ }^{5}$ skin, ${ }^{6}$ and nerve regeneration. ${ }^{7,8}$ An emerging trend in designing such scaffolds is recapitulating the natural extracellular matrix (ECM) traits by installing motifs capable of providing biochemical stimuli. ${ }^{9,10}$

Functionalization of alginate hydrogels is often achieved by covalently attaching bioactive molecules (e.g., peptides, proteins, etc.) to the alginate backbone. ${ }^{11-13}$ The pioneering work in creating alginate synthetic ECMs focused on binding single peptides to the alginate backbone, the first being the $\mathrm{G}_{4}$ RGDS peptide $;^{13}$ nowadays, researchers focus on creating multifunctional hydrogels by functionalizing the alginate with more than one type of biological molecule. ${ }^{14}$

\footnotetext{
${ }^{a}$ Department of Chemical Engineering, Ben-Gurion University of the Negev, Beer-Sheva 84105, Israel. E-mail: rbitton@bgu.ac.il

${ }^{b}$ Avram and Stella Goldstein-Goren Department of Biotechnology Engineering and Regenerative Medicine and Stem Cell Center, Ben-Gurion University of the Negev, POB 653, Beer-Sheva 84105, Israel

${ }^{c}$ Ilze Kats Institute for Nanoscale Science and Technology, Ben-Gurion University of the Negev, Beer-Sheva 84105, Israel

$\dagger$ Electronic supplementary information (ESI) available. See DOI: 10.1039/ d0ma00669f
}

The amount and identity of the attached biomolecules are crucial for the scaffold's bioactivity; however, they are not the only factors determining its functionality. The scaffold's stiffness as well as the ligand's availability (e.g., density) have been shown to dictate the magnitude and arrangement of intracellular forces, hence affecting the materials' cellular response to them. ${ }^{11,15,16}$ Therefore, understanding the structure-propertiesfunction relationship of multicomponent alginate hydrogels is an important step toward bettering their rational design.

In previous studies, we have shown that covalently linking a peptide that contains arginine-glycine-aspartic acid sequence (RGD) to an alginate backbone affected the conformational state of the individual chain as well as chain assemblies of alginate in aqueous solutions. ${ }^{17,18}$ We have shown that the amount of bound peptide determines the behaviour of polysaccharide-peptide conjugates in solution, regardless of the specific nature of the polysaccharide. ${ }^{19}$ Furthermore, we have demonstrated that the sequence of the conjugated peptides is a significant factor in tuning the stiffness of the alginate/peptide hybrid hydrogels. ${ }^{19}$

In this follow up study, we aim to explore the structureproperties-function relationship of multifunctional alginate hydrogels, i.e., hydrogels composed of an alginate chain decorated with more than one type of biomolecule. In particularly, we are interested in studying the effect of mode and order of conjugation on the resulting properties. To this end, we chose to covalently bind to the alginate backbone both the $\mathrm{G}_{4} \mathrm{RGDY}$ peptide and heparin. 
Heparin is a glycosaminoglycan (GAG), a stiff linear polysaccharide consisting of 1 to 4 linked disaccharide repeating units of uronic acid and glucosamine residues. ${ }^{20,21}$ Heparin has been shown to stabilize growth factors from denaturation while increasing the affinity of the complex to cell receptors, an important trait for tissue engineering applications. ${ }^{11-13} \mathrm{~A}$ variety of methods have been used for the covalent immobilization of heparin into hydrogels in the production of ECMmimetic materials. ${ }^{2,23}$ Recently, conjugation of heparin to polysaccharides (i.e.,) alginate ${ }^{14}$ and chitosan ${ }^{24}$ has been suggested to improve their performance as synthetic ECMs. ${ }^{14,15,25}$ It has been suggested that when designing a hydrogel for promoting cell adhesion, growth, and function, synthesizing a surface that contains both RGD and heparin would provide a more desirable biomimetic material than hydrogels containing only one of the individual components. ${ }^{26,27}$ However, optimizing the function and physical properties of these multicomponent hydrogels could be a rigorous process; a better understanding of the interplay between the building block's structure and the properties of the resulting hydrogels may be used to simplify it.

Here, we present a systematic study of the structuralphysical properties-function relationship of alginate$\mathrm{G}_{4} \mathrm{RGDY}$-heparin aqueous solutions and hydrogels. Using SAXS and rheology to characterize the systems' nanostructure, the viscosity of the solutions, and the storage modulus of the hydrogels, we show that attaching heparin to an alginatepeptide molecule greatly affects its physical properties. Moreover, we show that a detailed structural analysis of the conjugated architecture in solution can be used as a tool to adapt the properties of alginate-heparin-peptide hybrid hydrogels.

\section{Results and discussion}

\section{Synthesis of alginate-peptide-heparin}

Conjugation of a peptide and/or heparin to the alginate backbone was performed using carbodiimide chemistry as described in the experimental section. Overall, four modifications upon alginate were performed: conjugation of alginate with $\mathrm{G}_{4} \mathrm{RGDY}$ was termed as Alg-RGD. Conjugation of alginate with heparin was termed as Alg-Hep. Conjugating of Alg-RGD with heparin was termed as Alg-RGD-Hep, and conjugating of Alg-Hep with $\mathrm{G}_{4}$ RGDY was termed as Alg-Hep-RGD. The notation denotes the order of the conjugation and does not indicates the sequence (e.g. in Alg-RGD-Hep, heparin is not attached to RGD, but rather conjugated as the following step). In addition, a mixture of Alg-Hep and Alg-RGD (denoted as Alg-RGD + Alg-Hep) was prepared. The conjugation of the heparin and the peptide to the alginate was confirmed by FTIR spectroscopy (Fig. S1, ESI $\dagger$ ) and XPS (Table S1, ESI $\dagger$ ).

\section{Viscosity and nanostructure of modified alginate solutions}

Differences in the features of polymer network in solution are manifested in its' rheological behavior. Thus, the steady shear viscosity as a function of the applied shear rate was monitored for the polysaccharide's aqueous solutions. Viscosity measurements of $2 \mathrm{wt} \%$ polysaccharide (natural and modified) in aqueous solution are displayed in Fig. 1. As can be seen, all tested polymers exhibit shear thinning behaviour typical of viscous polymers at the semi-dilute concentration regime, ${ }^{18,21,28,29}$ in which a Newtonian plateau (low shear rate) region is followed by a viscosity gradual decrease (higher shear rate). The lowest shear rate at which the decrease of viscosity becomes apparent is called the critical shear rate $\left(\dot{\gamma}_{c}\right)$. Shear thinning of fluids and weak gels can be described by

$$
\eta=k \dot{\gamma}^{n-1}
$$

where $\eta$ is the viscosity ( $\mathrm{Pa} \mathrm{s}$ ), $\dot{\gamma}$ is the shear rate $\left(\mathrm{s}^{-1}\right), n$ is the power law exponent (for a Newtonian fluid $n=1$ ), and $k$ is a consistency index which is numerically equal to the viscosity at $1 \mathrm{~s}^{-1}$ (Table S2, ESI $\left.\dagger\right)^{30}$

As can be seen from Fig. 1, the behaviour of Alg-Hep is similar to that of alginate. This is expected due to the negative charge of the functional groups of both polymers, which prevents additional entanglements. In agreement with our previous work, ${ }^{17}$ Alg-RGD shows a much higher $k$ and a smaller $n$, indicating a more pronounced shear thinning behaviour (i.e., more entanglements). The scan of Alg-Hep-RGD lies between those of Alg-Hep and Alg-RGD, indicating both molecules are affecting the polymers' behaviour. Moreover, comparing the viscosity of Alg-RGD-Hep and Alg-Hep-RGD to the viscosity of a mixture of Alg-Hep and Alg-RGD (denoted as Alg-RGD + Alg-Hep) suggests a synergistic effect rather than an additive one, likely due to intermolecular interactions between Alg-RGD and Alg-Hep that are not substantial when the heparin and RGD are conjugated to the same alginate chain. The order of the molecules' conjugation (i.e., peptide before heparin or heparin before peptide) also had a significant effect on the solution's viscosity; fitting the data to a power-law model yielded higher $k$ and lower $\dot{\gamma}_{\mathrm{c}}$ (Table S2, ESI $\dagger$ ) for the molecules in which the peptide was the last conjugated molecule, suggesting a more entangled polymer network is formed. Contrarily, the $k$ of Alg-RGD-Hep is the lowest one.

Differences in the rheological behaviour of polymer solutions are usually caused by differences in the structural features of the polymer's network. Thus, SAXS measurements of the

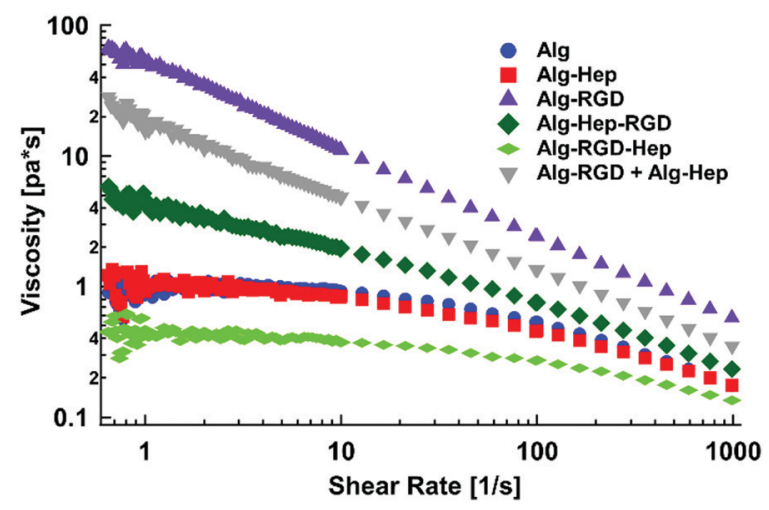

Fig. 1 Steady shear viscosity as a function of the applied shear rate of modified alginate in water (2 wt\%). 
modified alginate in aqueous solution were conducted. The SAXS patterns of all of the investigated molecules are presented in Fig. 2. The visible differences between the scattering curves indicate that indeed, there are structural differences between the tested solutions.

Horkay and Hammouda described the scattering pattern of polyelectrolyte in salt-free solutions as the result of three contributions: spatial concentration fluctuations (clustering), which are apparent as an upturn in the low $q$ range; thermal concentration fluctuations (high $q$ ); and a characteristic correlation peak (mid $q$ ) with a maximum, $q_{0}$, corresponding to $d_{0}=2 \pi / q_{0}$, which represents an average distance between the charged chains. ${ }^{31}$ This scattering pattern can be described by

$$
I(q)=\frac{A}{q^{n}}+\frac{C}{1+\left(\left|q-q_{0}\right| \zeta\right)^{m}}
$$

where $A$ and $C$ are constants, $n$ and $m$ are the clustering and solvation Porod exponents, respectively; $q_{0}$ is the peak position, if it can be resolved; and $\zeta$ is a correlation length that corresponds to an average distance between neighbouring entanglements within the same domain. ${ }^{31}$ The best fits to eqn (2) are presented as a black solid lines in Fig. 2, and the best-fit parameters are summarized in Table S3 (ESI $\dagger$ ).

As can be seen in Fig. 2, the most pronounced differences between the scattering curves are in the mid- and low $q$-regimes.
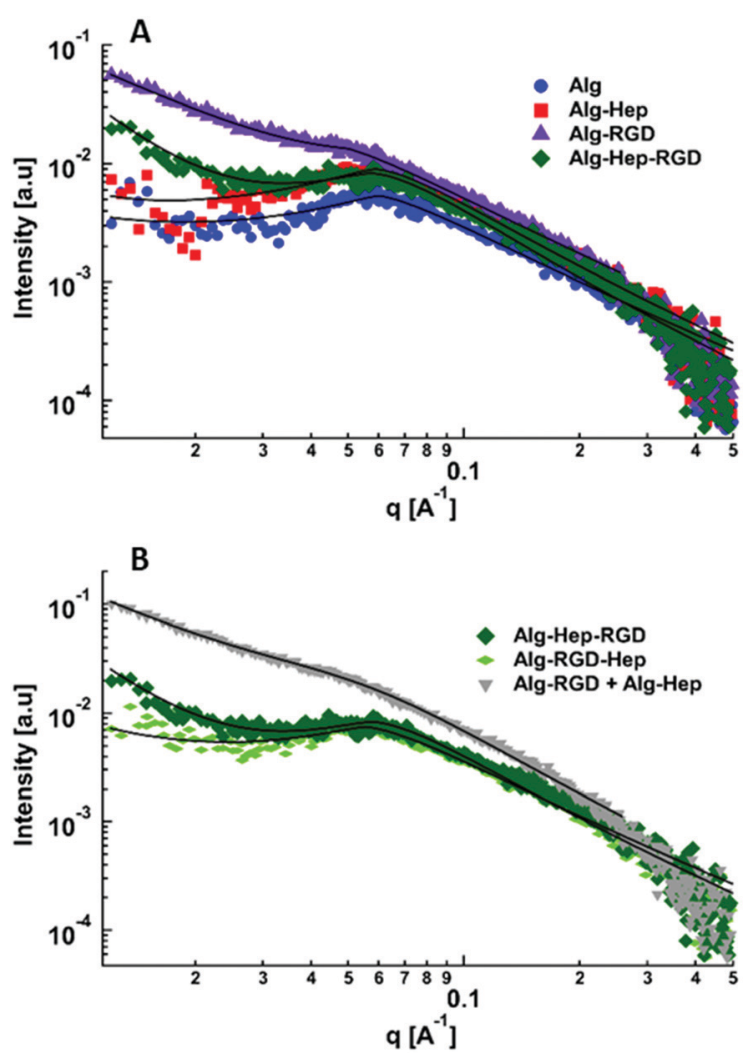

Fig. 2 Small-angle X-ray scattering curves of: (A) Alg, Alg-Hep, Alg-RGD, Alg-Hep-RGD, (B) Alg-Hep-RGD, Alg-RGD-Hep and Alg-RGD + AlgHep in water (2 wt\%). The curves were separated for better visualization. The black solid lines represent the best fits to eqn (2).
The characteristic correlation peak (mid- $q$ ) is clearly seen in the scattering pattern of alginate, Alg-Hep, Alg-Hep-RGD, and Alg-RGD-Hep; however, for Alg-RGD and Alg-RGD + Alg-Hep, while the correlation peak exists, it could not be well resolved. Fit of the data to eqn (2) shows that conjugating of both peptide and heparin to alginate chains did not significantly change the value of $d_{0}$. The larger $d_{0}$ value obtained for Alg-RGD + Alg-Hep is likely due to the fact that the correlation peak could not be well resolved and is not a true representation of the average distance between the charged domains (Table S3, ESI $\dagger$ ).

As seen by SAXS, the structural feature that is most affected by the alginate modification is the clustering. Alg-Hep-RGD, Alg-RGD, and the Alg-RGD + Alg-Hep mixture all exhibit a clear upturn in the low $q$ range. The clear distinction between the upturn and the correlation peak in the Alg-Hep-RGD curve is an indication that the clusters and the distance between the charged domains are of different length scales. The overlapping between the peak and the upturn in the scattering patterns of Alg-RGD and those of the Alg-RGD + Alg-Hep indicates the presence of clusters of various sizes, including some similar in size to $d_{0}$.

Both SAXS and viscosity measurements show that conjugating a peptide, heparin, or both to an alginate backbone affects the alginate's spatial organization in aqueous solutions. Moreover, the results suggest that the order of the peptide conjugation plays a significant role in determining the network's structure.

By comparing the results of shear-dependent viscosity to the SAXS patterns, one can see that the solutions with the higher viscosities exhibit an upturn in the low $q$ range of their scattering patterns. This observation implies that the higher viscosity of Alg-Hep-RGD, Alg-RGD + Alg-Hep, and Alg-RGD is due to the presence of large, dense clusters and not a smaller mesh size as would have been expected in the case of a homogeneous polymer network. Thus, we propose spatial organizations of heterogeneous networks, for the chain assemblies of modified alginates in aqueous solutions as depicted in Fig. 3.

Formation of large clusters is typically caused due to attractive inter-particle interactions. Large clusters are observed only for Alg-RGD, Alg-Hep-RGD and Alg-RGD + Alg-Hep, indicating that the peptide is imperative for their formation and that the attractive interactions are either between two $\mathrm{G}_{4}$ RGDY peptides or, more likely, between the peptide and the alginate backbone. Possible attractive interactions between these two molecules are hydrophobic interactions and hydrogen bonding, typical to polyphenol/polysaccharide interactions between the peptide's tyrosine and the alginate. ${ }^{17}$ However, for these interactions to take place, tyrosine needs to be accessible to the neighbouring molecules. In the case of Alg-RGD-Hep and Alg-Hep-RGD, one would expect the stronger electrostatic repulsion between the molecules, as a result of the presence of heparin, to prevent the formation of RGD-induced clusters. ${ }^{32}$ The fact that some clusters are formed in the latter (Alg-Hep-RGD) suggests that some of the peptides may have conjugated to the heparin instead of the alginate. Alg-Hep has a similar structure as unmodified alginate, since conjugation of heparin to the 

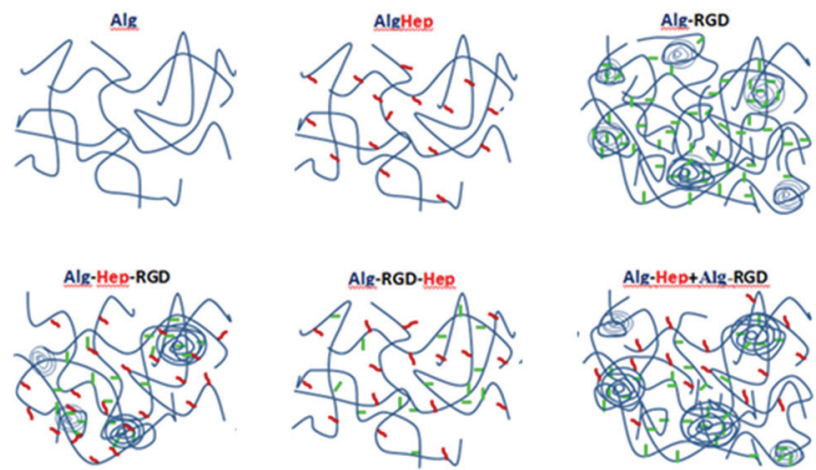

(2) cluster

$\sim$ Heparin

- RGD peptide

Alginate

Fig. 3 Illustration of the proposed spatial heterogeneous network organization of the modified alginate in aqueous solutions. The average distance between the charged domains is approximately the same for all the modifications. The structure of Alg-Hep is similar to that of Alg. The order of the peptide conjugation has a significant role on the tendency towards clustering; Alg-Hep-RGD, Alg-RGD + Alg-Hep, and Alg-RGD have large, dense clusters that are formed likely due to the attractive interactions either between two $G_{4}$ RGDY peptides or, more likely, between the peptide and the alginate backbone. The molecules are not drawn to scale.

alginate backbone did not affect the electrostatic repulsion between the chains, as heparin is also a polyanion.

\section{Mechanical properties and nanostructure of modified alginate hydrogels}

Next, hydrogels of modified alginate were prepared (as described in experimental section). Briefly, $\mathrm{CaCO}_{3}$ and GDL (to allow homogeny gels) were added to the alginate solutions so that the final concentrations were $1.5 \%$ wt $\%$ alginate, $40 \mathrm{mM} \mathrm{CaCO}_{3}$, and $40 \mathrm{mM}$ GDL. Rheological measurements were performed to quantify the mechanical properties (i.e., gel stiffness) of the different hydrogels. Frequency sweep scans of modified alginate hydrogels are presented in Fig. 4A. As expected from a gel's frequency sweep scan, both moduli $G^{\prime}$ and $G^{\prime \prime}$ are independent of the frequency of oscillation, and $G^{\prime}>G^{\prime \prime}$ (Fig. 4A); that is, the elastic nature of the sample is stronger than its viscous nature, as typically, gel stiffness is represented by its storage modulus $G^{\prime}$. A difference in $G^{\prime}$ values of the different gels is clearly apparent (Fig. 4B), indicating that conjugation of heparin and $\mathrm{G}_{4}$ RGDY affected the gels' mechanical properties, with Alg-RGD and Alg-RGD + Alg-Hep being much stiffer than the rest of the gels.

In previous studies of alginate-peptide gels, we attributed the increased stiffness of the gels to the presence of additional junction zones formed due to attractive interactions between the alginate and neighbouring peptides. ${ }^{17,33}$ The existence of these junction zones was revealed by SAXS, and more precisely by following the methodology of plotting the scattering curves of the alginate-peptide gels in a Kratky form, ${ }^{34}$ where a peak represents the presence of frozen inhomogeneities in the gel network. To identify whether the cause for the increased stiffness of the Alg-RGD and Alg-RGD + Alg-Hep also stems
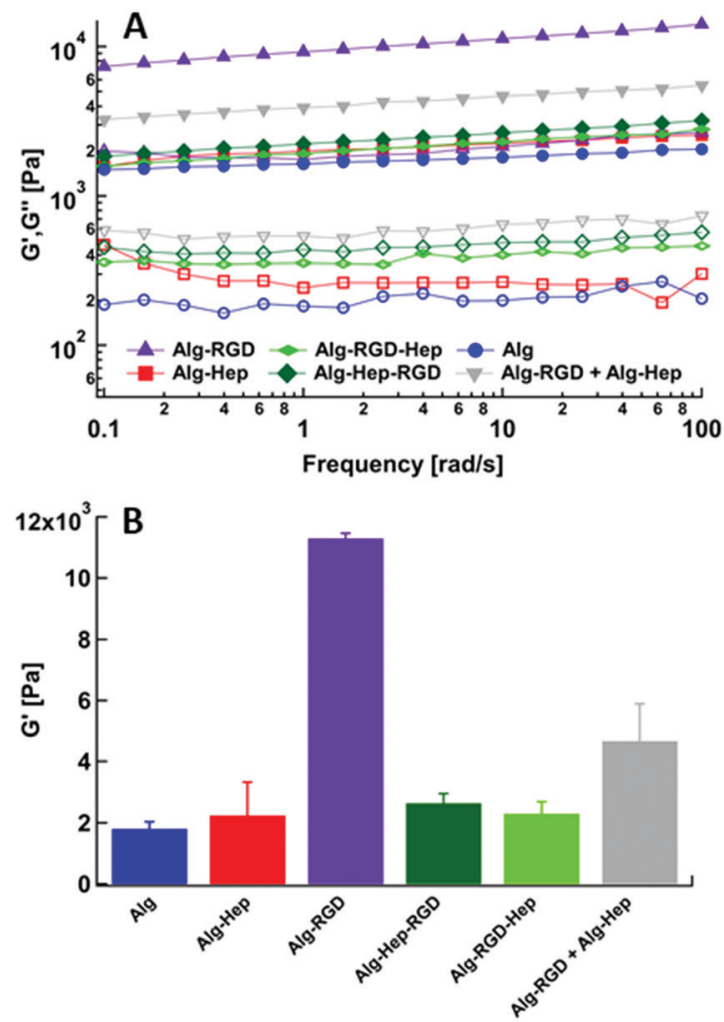

Fig. 4 (A) Frequency sweep results of modified alginate gels. G'(filled), $G^{\prime \prime}\left(\right.$ empty). (B) Storage modulus $\left(G^{\prime}\right)$ of modified alginate gels at frequency of $10 \mathrm{rad} \mathrm{s}^{-1}$.

from the presence of additional/larger junction zones, we performed SAXS measurements and presented the scattering curves of all gels in a Kratky plot (Fig. 5).

The pronounced peak that is evident in the Alg-RGD gel scattering curve, is evidence of the existence of additional (non-calcium) larger junction zones. The relatively less pronounced peaks of Alg-Hep-RGD, Alg-RGD-Hep and Alg-Hep indicate the existence of some non-calcium junction zones. Yet, the absence of a distinct peak implies that these junction zones are relatively small and evenly distributed. ${ }^{13,35,36}$ The scattering curve of the Alg-RGD + Alg-Hep gels lies between

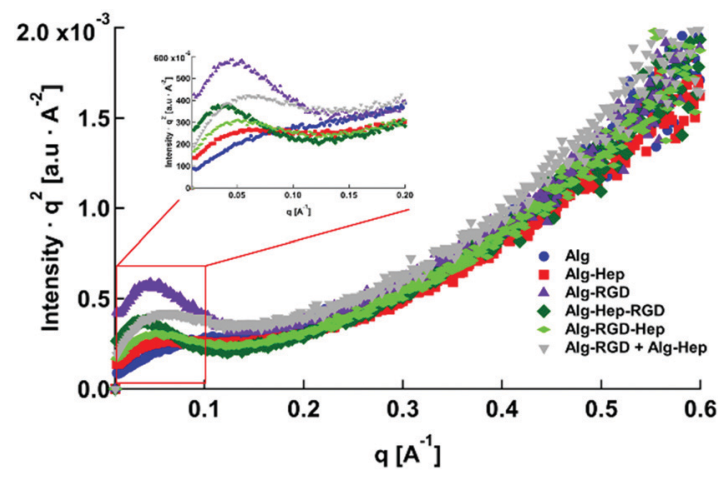

Fig. 5 Kratky plot of modified alginate hydrogels. The inset is an elragment of the peaks. 
that of Alg-Hep and that of Alg-RGD. Indeed, it is comparable to a mathematical addition of the scattering curves of Alg-RGD gel and Alg-Hep gel (Fig. S2, ESI $\dagger$ ), suggesting that the less pronounced peak of the Alg-RGD + Alg-Hep (compared to Alg-RGD) is not due to the presence of smaller non-calcium junction zones (compared to Alg-RGD), but rather due to an interpenetrating network structure in which the Alg-RGD and Alg-Hep gels coexist without affecting one another.

Not surprisingly, there is a correlation between the largest peak in the Kratky plot and the highest value of a gel's storage modulus. Though the conclusion of additional junction zones is similar to that of our previous study, it should be noted that the nature of these junction zones is different. $\mathrm{G}_{4}$ RGDY does not form micelles in water; thus, the junction zones are not similar to those suggested for Alg- $-\mathrm{V}_{6} \mathrm{KRGDY}$ or Alg- $\mathrm{A}_{6} \mathrm{KRGDY}$. Moreover, $\mathrm{G}_{4}$ RGDY is shorter and stiffer than the $\mathrm{G}_{6} \mathrm{KRGDY}$; thus, it does not disrupt the continuity of the $G$ blocks and hence does not prevent the formation of a large calcium junction zone. Here, the additional junction zones are most likely the clusters formed by the attractive interactions (described above) between the RGD peptide and the alginate backbone.

Taken together, our results thus far indicate that the structural features of the modified alginate chains as evident in solutions persist even after the gelation process, producing a correlation between the solution's and the gel's mechanical properties.

\section{Kinetics of vascular endothelial growth factor (VEGF) release}

It has been previously shown that structural features and mechanical properties of alginate hydrogels designed as ECM mimetics affect their functionality (i.e., bioactivity). ${ }^{37}$ The presence of heparin and/or the clusters within the hydrogels was expected to affect their ability to capture and release growth factors such as VEGF, due to its binding affinity to the heparin residues. ${ }^{26,38}$ Thus, in order to examine the structureproperties-function correlation in our system, we performed a preliminary study of the ability of the heparin containing gels to capture VEGF. VEGF was added to the modified alginate polymer solution and then gelation was induced (see Experimental section). VEGF molecules present in the medium during gelation, may or may not be captured in the gel, depending on the interactions between the VEGF molecules and the modified alginate polymer chains. ${ }^{39}$ In Fig. $6 \mathrm{~A}$ it can be seen that Alg-Hep, Alg-RGD-Hep and Alg-RGD + Alg-Hep gels captured the highest amounts of VEGF. These results indicate that the accessibility of the heparin, i.e. having heparin at the "end" of the attached molecule, is essential for the ability of the gel to form interactions with VEGF and as a result, to capture it. The highest amount of VEGF captured by Alg-RGD + Alg-Hep suggests that the presence of the clusters within the gel plays a secondary role in determining the gels' ability to capture VEGF, probably due to high local density of attractive interactions between the VEGF and alginate.

Next, we monitored VEGF release from the hydrogels during a period of 7 days (Fig. 6B). We were able to obtain VEGF release
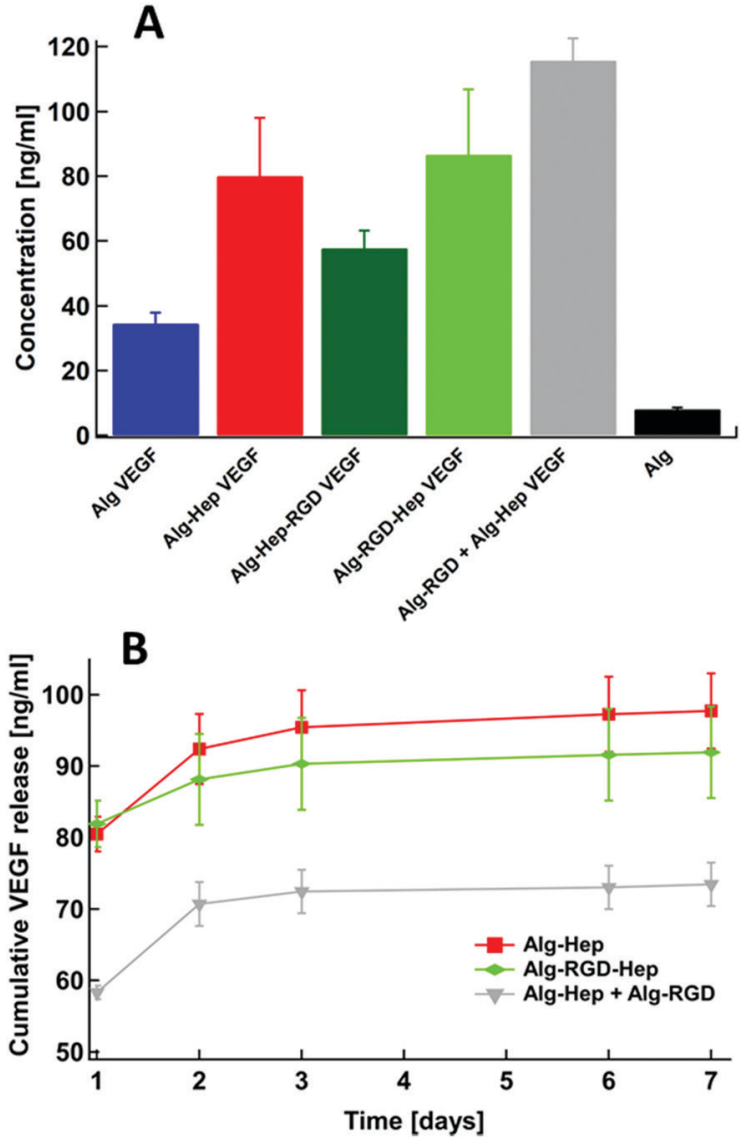

Fig. 6 (A) The initial VEGF amount in the hydrogels $(t=0)$; (B) release profiles of VEGF from the Alg-Hep gel, Alg-RGD-Hep gel, and Alg-RGD + Alg-Hep gel.

profiles only for Alg-Hep, Alg-RGD-Hep, and Alg-RGD + AlgHep gels (Fig. 6B) since the Alg and Alg-Hep-RGD hydrogels dissociated within $48 \mathrm{~h}$. A dissociation of ionically crosslinked alginate hydrogels in tissue culture medium is a well-known phenomenon and typically is attributed to the presence of calcium chelators, monovalent ions, and competing non-cross-linking divalent ions present in the external medium. ${ }^{40,41}$ Thus, the degree of hydrogel dissociation was visually monitored (Table S4, ESI $\dagger$ ). Alginate and Alg-Hep-RGD hydrogels, which had the lowest amount of captured VEGF, dissociated after $48 \mathrm{~h}$. The fact that the hydrogels with attached heparin at the "end" of the bound molecule were the most stable implies that the accessibility of heparin to interact with the surrounding molecules is imperative for these hydrogels' stability in medium.

Due to the dissociation of alginate and Alg-Hep-RGD hydrogels, VEGF release profiles were obtained only for Alg-Hep, Alg-RGD-Hep, and Alg-RGD + Alg-Hep gels (Fig. 6B). In all three gels, most of the encapsulated VEGF was released within 3 days. Comparing the release profiles of Alg-Hep and AlgRGD-Hep shows that the release of VEGF from Alg-Hep is slightly faster than from Alg-RGD-Hep (Fig. 6B). The extent and rate of dissociation for Alg-Hep and Alg-RGD-Hep seems to be the same (Table S4, ESI $\dagger$ ). Although Alg-RGD + Alg-Hep gel dissociates in a more pronounced manner comparing to the 
other two gels, it demonstrated lower cumulative VEGF release during 7 days. The high amount of VEGF captured in Alg-RGD + Alg-Hep as well as the lower release rate can be attributed to the presence of clusters in this gel. Diffusion of the growth factors through the clusters may be inhibited due to the high polymer density, explaining why even though the gel disintegrates, the release profile is very slow.

\section{Experimental}

\section{Materials}

Alginate (Protanal HF120RBS 55\% guluronate, sodium salt, $\left.\overline{M_{\mathrm{w}}}=425 \mathrm{kDa}\right)$ was a kind gift from FMC Biopolymers (Dramen, Norway). Heparin (183 USP units $\mathrm{mg}^{-1}, \overline{M_{\mathrm{w}}}=18 \mathrm{kDa}$ ), ethylenediamine (EDA, technical 75-80\%), $\mathrm{NaOH}, \mathrm{CaCO}_{3}$, 2 -[ $N$-morpholio] ethanesulfonic acid (MES buffer), $N$-hydroxysuccinimide sodium salt (NHS), (+)-gluconic acid $\delta$-lactone (GDL) and $N$-(3-dimetylaminopropyl)- $N$-ethylcarbodiimide hydrochloride (EDC) were purchased from Sigma-Aldrich. The peptide, $\mathrm{G}_{4}$ RGDY (80-90\% grade) was purchased from Bio-Sight/American Peptide Company (Vista, CA). Vascular endothelial growth factor (VEGF) and the ABTS EnzymeLinked Immune-Sorbent Assay (ELISA) Buffer Kit for VEGF were purchased from Peprotech Ltd.

\section{Methods}

Synthesis of polymer-heparin-peptide. Four molecules were synthesized: alginate- $\mathrm{G}_{4}$ RGDY (Alg-RGD); alginate-heparin (Alg-Hep); alginate-G ${ }_{4}$ RGDY-heparin (Alg-RGD-Hep); and alginate-heparin- $\mathrm{G}_{4}$ RGDY (Alg-Hep-RGD)

Synthesis of Alg-Hep conjugation of heparin to alginate was performed following the literature ${ }^{42}$ with some modifications. To minimize hydrolysis of EDC, the reaction was carried out in a $0.1 \mathrm{M}$ buffer of 2-morpholinoethane sulfonic acid (MES buffer). First, heparin sodium salt and sodium alginate (1:500 $\mathrm{mg} \mathrm{mg}^{-1}$ heparin : polymer) were dissolved in an MES buffer at a concentration of $1 \mathrm{wt} \%$ alginate with overnight stirring at room temperature. Then, the carboxylic groups of alginate and heparin were activated using EDC and NHS (molEDC: molNHS $=0.6: 1$ ). After $5 \mathrm{~min}, 1 \mathrm{~mL}$ of ethylenediamine $\left(3.1 \times 10^{-2} \mathrm{M}\right)$ was added to the solution under vigorous stirring at room temperature. After a reaction period of $4 \mathrm{~h}$, the resulting polymer was dialyzed to remove excess unreacted EDC/NHS and aminated heparin molecules, using a dialysis tubing cellulose membrane (14000 MWCO), against $1 \mathrm{M} \mathrm{NaCl}$ for $24 \mathrm{~h}$. The product was then frozen and lyophilized by freezedrier for $72 \mathrm{~h}$.

Synthesis of Alg-RGD conjugation of a peptide to the alginate was performed using carbodiimide chemistry, according to Rowley et al. ${ }^{13,43}$ Alginate was dissolved in the stock MES buffer $(0.1 \mathrm{M})$ to give a $1 \mathrm{wt} \%$ aqueous solution followed by stirring for $24 \mathrm{~h}$. NHS was added in an amount that would give a ratio of NHS : EDC of $1: 2$. The peptide was added after $5 \mathrm{~min}$ (1:100 $\mathrm{mg} \mathrm{mg}^{-1}$ peptide : polymer), and the solution was then stirred continuously for $24 \mathrm{~h}$. Thereafter, the solution was dialyzed against triply distilled water in 3500 MWCO dialysis tubes for $96 \mathrm{~h}$ and then lyophilized for $72 \mathrm{~h}$.

Synthesis of Alg-RGD-Hep was conducted by conjugating heparin to an Alg-RGD molecule rather than to pristine alginate.

Synthesis of Alg-Hep-RGD was conducted by conjugating $\mathrm{G}_{4} \mathrm{RGDY}$ to an Alg-Hep molecule instead of to pristine alginate.

For analysis, the requisite amount of the dried conjugated polymer was dissolved in triply distilled water $(18.2 \mathrm{M} \Omega \mathrm{cm})$.

Using carbodiimide chemistry could result in additional undesirable outcomes besides binding of the heparin and the peptide to the alginate backbone. These include: binding of heparin directly to the RGD peptide on the modified alginate chain and vice versa; formation of other derivatives in addition to the desired one (e.g. Alg-RGD, Alg-Hep, Alg-Alg, Hep-Hep, Hep-RGD); crosslinking reactions during modification of alginate with Hep to create Alg-Hep-Alg; and derivatives with noncomparable amounts of each component. However, the amount of those compounds will be considerably lower than the desired product.

Viscosity measurements. Viscosities of the polysaccharide solutions were determined using a HAAKE RotoVisco 1 (Thermo Scientific), equipped with an extended temperature cell for temperature control and a stainless steel cone-and-plate $\left(d=60 \mathrm{~mm}\right.$ and $\left.\theta=0.5^{\circ}\right)$. The viscosities of the polysaccharide solutions were measured at constant room temperature $\left(22 \pm 1{ }^{\circ} \mathrm{C}\right)$ as a function of the shear rate in an upward sweep from $1 \mathrm{~s}^{-1}$ to $1000 \mathrm{~s}^{-1}$. For these measurements, the alginate solutions were prepared by dissolving the alginate powder in Milli-Q water so the final concentrations were $2 \mathrm{wt} \%$ alginate.

Gel preparation. Alginate was dissolved in double-distilled water; modified alginate concentration in the gel was $1.5 \mathrm{wt} \%$. A calcium source in the form of a pre-prepared $40 \mathrm{mM} \mathrm{CaCO}_{3}$ solution was introduced into the alginate solution, followed by a fresh $\mathrm{D}-(+)$-gluconate- $\delta$-lactone (GDL) solution to induce gelation. $\mathrm{CaCO}_{3}$ has very low solubility in pure water, allowing its uniform distribution in alginate solution at neutral $\mathrm{pH}$. The addition of $\mathrm{D}-(+)$-gluconate- $\delta$-lactone to the alginate $/ \mathrm{CaCO}_{3}$ mixture induces the dissociation of $\mathrm{Ca}^{2+}$ from the $\mathrm{CaCO}_{3}$ by lowering the $\mathrm{pH}$. The released $\mathrm{Ca}^{2+}$ subsequently initiates the gelation of the alginate solution in a more gradual manner. ${ }^{15}$ The $\mathrm{Ca}^{2+}$ :GDL molar ratio was $1: 1$. The volume of the three solutions (alginate, $\mathrm{CaCO}_{3}$ and GDL) was designed to give a final volume of $0.5 \mathrm{~mL}$. The hydrogels were prepared as discs in a 48 well plate (11 $\mathrm{mm}$ diameter). The gelling solutions were allowed to rest in room temperature to 12 hours in humid conditions before Rheology and SAXS measurements were performed, to allow for the completion of the gelation process. ${ }^{44}$

Small angle X-ray scattering (SAXS). Small angle X-ray scattering patterns of the polymer solutions and hydrogels were obtained with a SAXSLAB GANESHA 300-XL. CuK $\alpha$ radiation was generated by a Genix 3D Cu-source with an integrated monochromator, 3-pinhole collimation and a two-dimensional Pilatus $300 \mathrm{~K}$ detector. The scattering intensity $q$ was recorded at a range of $0.012<q<3 \AA^{-1}$ (corresponding to lengths of 10-800 $\AA$ ). Measurements were performed under vacuum at 
ambient temperature. The scattering curves were corrected for counting time and sample absorption. Hydrogel specimens were placed in stainless steel sample cells with entrance and exit windows made of mica. Data analysis was based on fitting the scattering curve to an appropriate model by software provided by NIST (NIST SANS analysis version 7.0) on $\operatorname{IGOR}^{45}$ and model plot. ${ }^{46,47}$

Rheology. The dynamic viscoelastic properties of the modified alginate gel samples were characterized by determining the frequency dependence of the storage and loss moduli, $G^{\prime}(\omega)$ and $G^{\prime \prime}(\omega)$ at $T=25{ }^{\circ} \mathrm{C}$ after inducing the gelation. Prior to these measurements, strain sweep experiments were performed in order to establish the linear viscoelastic region of the hydrogels, and a strain of $1 \%$ was chosen for frequency sweep measurements.

Measurements were carried out with a Reologica StressTech HR stress-controlled rheometer equipped with an extended temperature cell for temperature control and stainless steel parallel plate $(d=8 \mathrm{~mm})$.

Release studies and VEGF analysis by enzyme linked immuno-sorbent assay (ELISA). $1.0 \mu \mathrm{L}$ from $1 \mu \mathrm{g} \mu \mathrm{L}^{-1}$ VEGF stock solution was added to $1 \mathrm{~mL}$ modified alginate solutions $1.5 \mathrm{wt} \%$ and incubated for $2 \mathrm{~h}$ at $37{ }^{\circ} \mathrm{C}$ in incubator under stirring. Next, $\mathrm{CaCO}_{3}$ was added to a final concentration of $40 \mathrm{mM}$. The solution was transferred to 96 well-plate, $100 \mu \mathrm{L}$ for each well and then GDL was added to each well to a final concentration of $40 \mathrm{mM}$. The solutions were incubated at RT for $24 \mathrm{~h}$ to allow the completion of the gelling process.

For the determination of initial amount of VEGF at each gel $(t=0)$, the gels were dissociated by adding $300 \mu \mathrm{L}$ of Sodium citrate $6 \%$ in PBS and $600 \mu \mathrm{L}$ Dulbecco's modified eagle medium (DMEM) with $0.1 \%$ bovine serum albumin (BSA, $\mathrm{pH}$ 7.2). The content of each well was transferred to low protein binding (LB) eppendorfs $(1.5 \mathrm{~mL})$ and stored at $-80{ }^{\circ} \mathrm{C}$ until the ELISA was done.

For the release experiments, the modified alginate gels containing VEGF were placed in DMEM with 0.1 wt $\%$ BSA, pH 7.2 and incubated on an incubator at $37{ }^{\circ} \mathrm{C}$. The supernatant was collected and replaced by fresh DMEM with $0.1 \%$ BSA at scheduled time points and kept frozen at $-80{ }^{\circ} \mathrm{C}$ until all samples were collected and the amount of VEGF in releasing media was determined by an ELISA. The ELISA was performed following the manufacturer's instructions (Human VEGF165 Standard ABTS ELISA Development Kit, ABTS ELISA Buffer Kit, Peprotech Ltd).

\section{Conclusions}

We explored the conjugation manner of alginate modified with both heparin and the $\mathrm{G}_{4}$ RGDY peptide. SAXS and rheology measurements showed that large clusters were formed only in the systems in which RGD was accessible to easily interact with a neighbouring alginate backbone (i.e., Alg-RGD and Alg-RGD + Alg-Hep). These large clusters also led to higher viscosity and a more pronounced shear thinning behavior of those solutions, indicating that the order of heparin and peptide conjugation to the alginate backbone plays a significant role in determining the structural-mechanical property relations of these modified alginate solutions.

SAXS of the modified alginate gels shows that the large clusters formed in solutions remained intact during the calcium-induced gelation process, leading to gels with a higher storage modulus and slower release rates of the VEGF incorporated within them. Therefore, the mode and order of conjugating different molecules to the alginate backbone should be considered when designing such multicomponent alginate hydrogels. A detailed structural analysis of the conjugated architecture in solution can be used as a tool to adapt the properties of alginate-heparin-peptide hybrid hydrogels.

\section{Conflicts of interest}

There are no conflicts to declare.

\section{References}

1 T. Coviello, P. Matricardi, C. Marianecci and F. Alhaique, J. Controlled Release, 2007, 119, 5-24.

2 C. Borselli, C. A. Cezar, D. Shvartsman, H. H. Vandenburgh and D. J. Mooney, Biomaterials, 2011, 32, 8905-8914.

3 I. Freeman and S. Cohen, Biomaterials, 2009, 30, 2122-2131.

4 Y. M. Kolambkar, K. M. Dupont, J. D. Boerckel, N. Huebsch, D. J. Mooney, D. W. Hutmacher and R. E. Guldberg, Biomaterials, 2011, 32, 65-74.

5 R. Dittrich, G. Tomandl, F. Despang, A. Bernhardt, T. Hanke, W. Pompe and M. Gelinsky, J. Am. Ceram. Soc., 2007, 90, 1703-1708.

6 L. Shi, L. Xiong, Y. Hu, W. Li, Z. Chen, K. Liu and X. Zhang, Polym. Eng. Sci., 2018, 58, 1782-1790.

7 T. Hashimoto, Y. Suzuki, K. Suzuki, T. Nakashima, M. Tanihara and C. Ide, J. Mater. Sci.: Mater. Med., 2005, 16, 503-509.

8 D. Szarek, K. Marycz, P. Bednarz, P. Tabakow, W. Jarmundowicz and J. Laska, Biotechnol. Appl. Biochem., 2013, 60, 547-556.

9 Y. Sapir, O. Kryukov and S. Cohen, Biomaterials, 2011, 32, 1838-1847.

10 K. Uto, J. H. Tsui, C. A. DeForest and D.-H. Kim, Prog. Polym. Sci., 2017, 65, 53-82.

11 T. Andersen, P. Auk-Emblem and M. Dornish, Microarrays, 2015, 4, 133-161.

12 R. Censi, P. Di Martino, T. Vermonden and W. E. Hennink, J. Controlled Release, 2012, 161, 680-692.

13 J. A. Rowley, G. Madlambayan and D. J. Mooney, Biomaterials, 1999, 20, 45-53.

14 O. Jeon, C. Powell, L. D. Solorio, M. D. Krebs and E. Alsberg, J. Controlled Release, 2011, 154, 258-266.

15 O. Jeon, D. S. Alt, S. M. Ahmed and E. Alsberg, Biomaterials, 2012, 33, 3503-3514.

16 B. Trappmann and C. S. Chen, Curr. Opin. Biotechnol, 2013, 24, 948-953. 
17 G. Ochbaum and R. Bitton, Polymer, 2017, 108, 87-96.

18 O. Bernstein-Levi, G. Ochbaum and R. Bitton, Colloids Surf., $B, 2016,137,214-220$.

19 G. Ochbaum, M. Davidovich-Pinhas and R. Bitton, Soft Matter, 2018, 14, 4364-4373.

20 Y. Liang and K. L. Kiick, Acta Biomater., 2014, 10, 1588-1600.

21 D. L. Rabenstein, Nat. Prod. Rep., 2002, 19, 312-331.

22 T. Nie, R. E. Akins Jr and K. L. Kiick, Acta Biomater., 2009, 5, 865-875.

23 T. Nie, A. Baldwin, N. Yamaguchi and K. L. Kiick, J. Controlled Release, 2007, 122, 287-296.

24 S. Boddohi, N. Moore, P. A. Johnson and M. J. Kipper, Biomacromolecules, 2009, 10, 1402-1409.

25 A. D. Baldwin and K. L. Kiick, J. Pept. Sci., 2010, 94, 128-140. 26 M. M. Adil, T. Vazin, B. Ananthanarayanan, G. M. Rodrigues, A. T. Rao, R. U. Kulkarni, E. W. Miller, S. Kumar and D. V. Schaffer, Biomaterials, 2017, 136, 1-11.

27 A. Tiwari, H. J. Salacinski, G. Punshon, G. Hamilton and A. M. Seifalian, FASEB J., 2002, 16, 791-796.

28 G. N. Liberman, G. Ochbaum, S. M. Arad and R. Bitton, Carbohydr. Polym., 2016, 152, 658-664.

29 R. Tuinier, P. Zoon, M. C. Stuart, G. Fleer and C. De Kruif, Biopolymers, 1999, 50, 641-646.

30 J. F. Steffe, Rheological methods in food process engineering, Freeman press, 1996.

31 F. Horkay, P. J. Basser, A.-M. Hecht and E. Geissler, Phys. Rev. Lett., 2008, 101, 068301.

32 C. Rodríguez-Rivero, L. Hilliou, E. M. M. del Valle and M. A. Galán, Rheol. Acta, 2014, 53, 559-570.

33 G. Ochbaum, E. Chetrit, R. Berkovich and R. Bitton, Soft Matter, 2020, 16, 6155-6162.
34 Y. Maki, K. Ito, N. Hosoya, C. Yoneyama, K. Furusawa, T. Yamamoto, T. Dobashi, Y. Sugimoto and K. Wakabayashi, Biomacromolecules, 2011, 12, 2145-2152.

35 E. M. Saffer, M. A. Lackey, D. M. Griffin, S. Kishore, G. N. Tew and S. R. Bhatia, Soft Matter, 2014, 10, 1905-1916.

36 J. Zaragoza, N. Babhadiashar, V. O'Brien, A. Chang, M. Blanco, A. Zabalegui, H. Lee and P. Asuri, PLoS One, 2015, 10(8), e0136293.

37 M. Ahearne, Interface Focus, 2014, 4, 20130038.

38 U. Freudenberg, A. Hermann, P. B. Welzel, K. Stirl, S. C. Schwarz, M. Grimmer, A. Zieris, W. Panyanuwat, S. Zschoche and D. Meinhold, Biomaterials, 2009, 30, 5049-5060.

39 Y. Shmidov, M. Zhou, G. Yosefi, R. Bitton and J. B. Matson, Soft Matter, 2019, 15, 917-925.

40 A. Martinsen, G. Skjåk-Bræk and O. Smidsrød, Biotechnol. Bioeng., 1989, 33, 79-89.

41 M. A. LeRoux, F. Guilak and L. A. Setton, J. Biomed. Mater. Res., 1999, 47, 46-53.

42 Q. Zuo, R. Guo, Q. Liu, A. Hong, Y. Shi, Q. Kong, Y. Huang, L. He and W. Xue, Biomed. Mater., 2015, 10, 035008.

43 M. Daoud, J. Cotton, B. Farnoux, G. Jannink, G. Sarma, H. Benoit, C. Duplessix, C. Picot and P. De Gennes, Macromolecules, 1975, 8, 804-818.

44 B. Polyak, S. Geresh and R. S. Marks, Biomacromolecules, 2004, 5, 389-396.

45 S. R. Kline, J. Appl. Crystallogr., 2006, 39, 895-900.

46 B. T. Stokke, K. I. Drager, Y. Yuguchi, H. Urakawa and K. Kajiwara, Macromol. Symp., 1997, 120(1), 91-101.

47 B. T. Stokke, K. I. Draget, O. Smidsrød, Y. Yuguchi, H. Urakawa and K. Kajiwara, Macromolecules, 2000, 33, 1853-1863. 